\title{
Downlink packet scheduling and resource allocation in EPON WiMAX hybrid access networks
}

\author{
Hung-Yi Teng, Chung-Chih Kuo and Ren-Hung Hwang ${ }^{*}$
}

\begin{abstract}
Multimedia applications over the Internet, such as IPTV and video-on-demand, have become fast growing applications in recent years. Such applications have stringent quality of services (QoS) constraints in terms of bandwidth, delay, and packet loss. As a consequence, broadband access networks play an important role for multimedia applications. There are two emerging technologies offering both high bandwidth and QoS support, namely Ethernet Passive Optical Network (EPON) and Worldwide Interoperability for Microwave Access (WiMAX). By integrating these two technologies, EPON-WiMAX integrated network can: (1) provide broadband access, (2) support mobile users, and (3) decrease network planning cost and operating cost. Thus, EPON-WiMAX integrated network is an ideal choice for multimedia applications with ubiquitous access. Although EPON-WiMAX integrated network has received growing attentions, however, most of previous works focus on the scheduling and bandwidth allocation in the upstream direction. Therefore, in this paper, we investigate the downlink scheduling and bandwidth allocation problem in EPON-WiMAX integrated networks. The objective of the study is to maximize the system throughput and guarantee the (QoS) so that the requirements of multimedia applications can be fulfilled. We proposed a two-stage downlink packet scheduling and resource allocation mechanism collaborating with application layer forward error correction (AL-FEC). We demonstrated the performance of our approach via simulations. Our simulation results indicated that the proposed mechanism increased the system throughput significantly, especially when AL-FEC is adopted.
\end{abstract}

Keywords: EPON-WiMAX integrated network, Downlink, Packet scheduling, Resource allocation, Application layer forward error correction (AL-FEC)

\section{Introduction}

Recently, a variety of popular multimedia applications have posted a high bandwidth requirement, such as high-definition television and video-on-demand services, which indicated that the broadband access technology has become more and more important. In wired networks, Ethernet Passive Optical Network (EPON) [1] adopts optical fiber as the transmission medium. EPON is a point-to-multipoint fiber access network which supports up to $10 \mathrm{Gbps}$ bandwidth. Since it can provide high bandwidth and is compatible with legacy Ethernet, it is considered as one of the solutions for the next generation wired broadband access technologies. In wireless networks, Worldwide Interoperability for Microwave Access (WiMAX) $[2,3]$ is a new generation of broadband

\footnotetext{
* Correspondence: rhhwang@cs.ccu.edu.tw

Department of Computer Science and Information Engineering, National Chung-Cheng University Chiayi, Taiwan, Republic of China
}

wireless access technology which supports long-distance, high-bandwidth, quality of service QoS-guaranteed wireless communications. Therefore, it has been identified as one of the last mile solutions. Although EPON and WiMAX technologies are promising, it should be noted that deploying EPON or WiMAX still has some limitations. For example, deploying Fiber To The Home is expensive for Internet Service Providers. On the other hand, the data-transfer rate of mobile WiMAX subscriber stations (SSs) in current real-world implementation may only be maxing out around $70 \mathrm{Mbps}$ over a $20 \mathrm{MHz}$ channel which is much less than that of wired networks.

Therefore, Shen et al. [4] proposed the integration of EPON and WiMAX networks to make up for each other's deficiencies. Advantages of the integrated EPON-WiMAX network include providing broadband Internet access, supporting mobile users, and reducing 
the network design and maintenance costs. In general, the architecture of the EPON-WiMAX network is a tree topology, consisting one EPON Optical Line Terminal (OLT), one 1:N passive optical splitter, and multiple EPON Optical Network Units (ONUs) which consequently connects to a WiMAX Base Station (BS). Based on how EPON ONU and WiMAX BS are constituted, the architecture can be classified into four categories, namely independent, hybrid, unified, and microwaveover-fiber. Among these four architectures, the hybrid architecture is the most promising with advantages of more flexible development, less deployment costs, and less technology restrictions. In the hybrid architecture, EPON ONU and WiMAX BS are integrated into one device logically or physically, referred to as ONU-BS. An ONU-BS consists of three components: EPON ONU, WiMAX BS and a central control unit. The central control unit is responsible for conversion between EPON and WiMAX networks, such as frame format conversion and QoS mapping. Traffic between OLT and ONU is transmitted on two separated wavelengths, typically $1310 \mathrm{~nm}$ (for upstream) and $1550 \mathrm{~nm}$ (for downstream). A MultiPoint Control Protocol (MPCP) is used as a control and signaling mechanism between OLT and ONU which is specified in the IEEE 802.3ah standard [5].

For the integration of EPON and WiMAX, packet scheduling and bandwidth allocation in upstream direction have received much more attention. Yang et al. [6] presented a converged network architecture based on the concept of virtual ONU-BS (VOB) and proposed a QoS-aware dynamic bandwidth allocation (DBA) scheme. The proposed QoS-aware DBA scheme is operated in a hierarchical manner and therefore can support bandwidth fairness at the VOB level as well as class of traffic fairness at the SS level. Jung et al. [7] investigated three possible integrated architectures and proposed a centralized scheduling (CS) mechanism to enhance end-to-end delay and provide better QoS provisioning for the lower priority traffic. Relatively less attention has been focused on downlink packet scheduling and bandwidth allocation on the integrated EPON WiMAX network. Emphasizing on inter-cell cooperative transmission, Gong et al. [8] proposed three schemes to optimize ONU-BS user association and resources allocation (BUA-RA) in terms of minimizing the number of rejected connection requests. However, QoS guarantee for different classes of traffic was not considered, which is essential in mobile WiMAX [9].

In this study, a two-stage design of downlink packet scheduling and resources allocation was proposed for the EPON-WiMAX hybrid access networks. In the firststage, packet scheduling from OLT to ONU-BSs was considered to balance the load among ONU-BSs to ensure each ONU-BS had abundant resources to transmit packets to its SSs. In the second-stage, packet scheduling from ONU-BS to SSs was considered. To provide end-to-end bounded-delay and fair allocation of bandwidth, $\mathrm{WF}^{2} \mathrm{Q}$ [10] was adopted to schedule packets to be transmitted on each downlink sub-frame. Since channel condition can vary considerably across users in a wireless environment, packet loss is one of the main obstacles to fulfill QoS of traffic classes. To enhance end-to-end reliability and resources utilization of each ONU-BS, a combination of application layer forward error correction (AL-FEC) [11] and modulation and coding scheme (MCS) was proposed. As a result, the overall system throughput was improved and packet transmission delay was reduced while QoS of each traffic class could be guaranteed. The performance of our approach is demonstrated via simulations. The simulation results indicated that the proposed mechanism increased the overall system throughput significantly while guaranteeing the QoS of traffic classes, especially when AL-FEC is adopted.

The rest of the article is organized as follows. We first present a literature survey on EPON-WiMAX integrated networks in Section 2. Section 3 gives an overview of the EPON WiMAX architecture, WiMAX handover schemes, and problem formulation. In Section 4, we proposed a two-stage scheduling algorithm and the use of AL-FEC to improve the system throughput while guaranteeing the QoS of each traffic class. Simulation results are presented in Section 5. Finally, conclusion and future work are given in Section 6.

\section{Related works}

For offering wider bandwidth and mobility with low costs to users, much research effort has been conducted on the integration of EPON and WiMAX. Shen et al. [4] proposed four architectures for integration of EPON and WiMAX, namely independent, hybrid, unified, and microwave-over-fiber, in which related research issues are elaborated. Ghazisaidi and Maier [12] proposed a techno-economic model to evaluate the cost-performance trade-offs of EPON and WiMAX networks. An optimal ONU-BS placement model was presented in [13], which jointly take BS-User association and resource breakdown assignment into consideration. Kim et al. [14] proposed a distributed antenna based EPON-WiMAX integration architecture with a cost-efficient cell planning mechanism which was used to optimally control the size of overlapped cell coverage areas.

For integration of EPON and WiMAX, packet scheduling and bandwidth allocation in upstream direction have received much more attention. Yang et al. [6] presented a converged network architecture based on the concept of VOB which is a logical form of the hybrid architecture presented in [4]. The authors also proposed a QoS-aware DBA scheme which can support bandwidth 
fairness at the VOB level and class of traffic fairness at the SS level. Jung et al. [7] investigated three possible integrated architectures: independent ONU-BS, combined ONU-BS, and hybrid ONU-BS. To address the problem of the independent scheduling, the authors proposed a CS mechanism to enhance end-to-end delay and provide better QoS support. Ranaweera et al. [15] investigated different DBA algorithms, intra ONU-BS scheduling algorithms, and QoS mapping mechanisms on the QoS performance of the EPON-WiMAX converged network. A hybrid priority weighted fair scheduling was also proposed to avoid bandwidth starvation in the lower priority traffic classes. Dias Piquet and Saldanha Fonseca [16] assessed the performance of a standard-compliant WiMAX uplink scheduler and showed that the scheduler can provide QoS support to the SSs.

Relatively less attention has been focused on downlink packet scheduling and bandwidth allocation on the integration of EPON and WiMAX. In [17], a cross-layer design for video multicasting was proposed, where a modified MDC on scalable video streams at the application layer, superposition coding at physical layer as well as inter-cell cooperative transmission are jointly considered. Emphasizing on inter-cell cooperative transmission, Gong et al. [8] proposed three schemes to optimize BUA-RA in terms of minimizing the number of rejected connection requests and time slot usage. However, this study cannot provide QoS guarantee of different traffic classes. Therefore, in this study, we proposed two-stage design of downlink packet scheduling and resources allocation to improve the system throughput and provide QoS guarantee for each traffic class.

\section{System model}

\subsection{Network environment}

As shown in Figure 1, there are three main components in an integrated EPON-WiMAX network. The OLT, a component of EPON, receives packets from the wired network and then transfers them to ONU-BSs. The ONU-BS is an integrated component of the ONU of EPON and the BS of WiMAX. It receives packets from the EPON wired network and then transmits them to SSs through the WiMAX network. The SS is a component of WiMAX which receives packets only from the ONU-BS. In this study, we focused on the downlink packet scheduling problem at the OLT and ONU-BS. Thus, in our integrated EPON-WiMAX network environment, we only investigated the scenarios of one OLT. We assumed that each ONU-BS was able to access full context of all SSs which are under its coverage, including distance and signal strength. It passes the information of SS to OLT using MPCP. We also assumed that each SS can associate with only one BS as its serving BS at any given moment although an SS could be within the transmission range of two or more ONU-BSs, as shown in Figure 1. Furthermore, an SS could be a mobile station and its SINR value varies from time-to-time. Therefore, an SS could handoff from one ONU-BS to another.

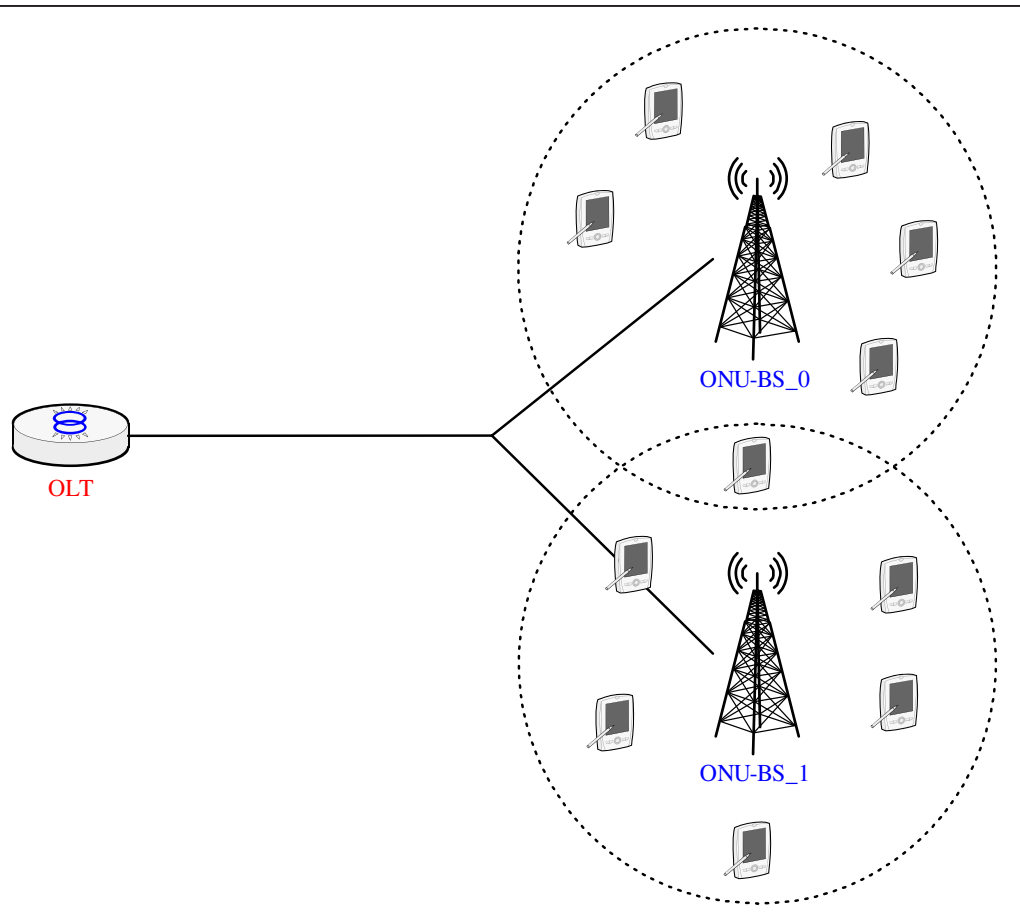

Figure 1 EPON-WiMAX network topology. 
There are three handoff mechanisms supported in IEEE 802.16e, namely, Hard Handover, Macro-Diversity Handover (MDHO), Fast Base Station Switching (FBSS). For delay-sensitive applications, MDHO and FBSS are better mechanisms [18]. In this study, we assumed that the FBSS mechanism was adopted because of its flexibility. Coordinated by OLT, the serving ONU-BS can assist its SSs to associate the designated ONU-BS for better network throughput.

\subsection{Problem formulation}

In an integrated EPON WiMAX network, we divided the SSs into two categories: determined SS (DSS) and overlapped SS (OSS). A DSS was under the coverage of only one ONU-BS and only one serving BS can be selected. Whereas an OSS was covered by more than two ONU-BS, so that it can select one of the ONU-BSs as its serving BS. Our goal was to optimize the aggregated network throughput via the scheduling algorithm at the OLT. Since the OLT knows the context of all SSs, two factors were considered by the scheduling algorithm. First, for each OSS, the scheduler decided through which ONU-BS to send packets such that the network throughput could be maximized. Secondly, for each SS, the scheduler adaptively selected the combination MCS and AL-FEC code $[9,19]$ that the network throughput could be maximized. Note that although the MCS and AL-FEC codes could be determined at the transmitting ONU-BS, however, global optimization could be better achieved if they were chosen at the OLT. Consequently, our problem was formulated into how does the OLT select the serving ONU-BS for each OSS and the MCS and FEC for each SS on behalf of the serving ONU-BS, in that the aggregated network throughput could be maximized while the packet loss rate is guaranteed to meet the requirement of the WiMAX standard. Our objective function is as follows:

$$
\underset{T S_{m n}, A_{m n}, M C S_{n}, F_{n}}{\operatorname{Maximize}} \sum_{n=1}^{N} R_{n}
$$

Subject to:

$$
\begin{gathered}
\sum_{n=1}^{N} T s_{m n} A_{m n} \leq T S S_{m}, \forall m=1 \ldots M \\
\sum_{m=1}^{M} A_{m n}=1, \forall n=1 \ldots N \\
R_{n}=M C S_{n} \times F_{n} \times \sum_{m=1}^{M} T s_{m n} A_{m n} \geq R_{n}^{r e q}, \forall n=1 \ldots N \\
R_{n}^{r e q}=R_{n}^{U G S \_r e q}+R_{n}^{r t P S \_r e q}+R_{n}^{n r t P S \_r e q}
\end{gathered}
$$

$$
\begin{aligned}
& R_{n}^{U G S \_r e q}=\sum_{j \in U G S} M R R_{j}^{r e q}, \forall j=1 \ldots J \\
& R_{n}^{r t P S_{-} r e q}=\max \left(\sum_{j \in r t P S} M R R_{j}^{\text {req }}, \sum_{j \in r t P S} M L T R_{j}^{r e q}\right) \\
& \forall j=1 \ldots J \\
& R_{n}^{\text {nrtPS_req }}=\sum_{j \in \text { rttPS }} M R R_{j}^{\text {req }}, \forall j=1 \ldots J \\
& A_{m n}= \begin{cases}1, & \text { if } \text { ONU }-B S_{m} \text { associates } S S_{n} \\
0, & \text { otherwise }\end{cases} \\
& \operatorname{GER}_{n}\left(S_{N} R_{n}, M_{C} S_{n}, F_{n}\right)<\text { Threshold } \text { loss_rate }
\end{aligned}
$$

Equation (1) aims to maximize the sum of individual received bandwidth (throughput) $\left(R_{n}\right)$ of each SS assuming that there are $M$ ONU-BSs and $N$ SSs. But it is subjected to the restrictions of Equations (2)-(10). Equation (2) indicates that for each ONU-BS $(m)$, the number of the time slots $\left(T s_{m n}\right)$ that are allocated to the SS does not exceed the total number of time slots that are available $\left(T S S_{m}\right)$. Equation (3) indicates that for each SS (n), it can select only one ONU-BS as its serving BS at any given moment. Equation (4) indicates that for all the $S S_{n}$, the bandwidth $\left(R_{n}\right)$ each SS is allocated must satisfy its minimum required bandwidth $\left(R_{n}^{r e q}\right)$, i.e., its QoS requirement. Note that $M C S_{n}$ is the MCS selected for $S S_{n}$ for receiving packets, in bits per slot; $F_{n}$ is the code rate of the error correction code selected for $S S_{n}$; $\sum_{m=1}^{M} T s_{m n} A_{m n}$ is the total number of time slots allocated to the $S S_{n}$. The required bandwidth $\left(R_{n}^{r e q}\right)$ of $S S_{n}$ is calculated using equations (5) and (8). Equation (5) indicates that the required bandwidth $\left(R_{n}^{r e q}\right)$ of $S S_{n}$ is the sum of bandwidth required for each QoS class, namely, UGS, rtPS, and nrtPS. Equations (6) and (8) indicate that bandwidth required for UGS and nrtPS traffic are the sum total of the minimum required rate $\left(M R R_{j}^{r e q}\right)$, respectively. Equation (7) indicates that bandwidth required for the rtPS traffic is the greater value of the sum total of the minimum required rate $\left(M R R_{j}^{r e q}\right)$ or the sum total of the bandwidth $\left(M L T R_{j}^{r e q}\right)$ with which the system would finish transmitting all the packets by the requested deadline. Equation (9) indicates that $A_{m n}$ is a $0 / 1$ variable where 1 and 0 represents that ONU-BS ${ }_{\mathrm{m}}$ is and is not the serving BS of $S S_{n}$, respectively. Equation (10) indicates that through our appropriate selection of $M C S_{n}$ and $F_{n}$, the packet loss rate $\left(G R_{n}\right)$ of $S S_{n}$ will be within the threshold of the WiMAX standard. Table 1 shows the notation used in this study.

To solve this problem, we proposed a two-phase solution in the following section. In the first phase, we solved the formula (10) by the proposed MCS and FEC selecting algorithm MFSA algorithm that determines which combination of the MCS and AL-FEC was the 


\begin{tabular}{|c|c|}
\hline Symbol & Meaning \\
\hline$M$ & The total number of ONU-BS in the system \\
\hline N & The total number of SS in the system \\
\hline J & The maximum number of connections per SS \\
\hline$T_{S S}$ & The maximum resource (time slots) of ONU-BS $m$ \\
\hline$T s_{m n}$ & $\begin{array}{l}\text { The resource (time slots) allocated to SS } n \\
\text { by ONU-BS } m\end{array}$ \\
\hline$A_{m n}$ & Whether ONU-BS $m$ is the serving BS of SS $n$ \\
\hline$R_{n}$ & The actual bandwidth (bps) allocated to SS $n$ \\
\hline$R_{n}^{r e q}$ & The minimum required bandwidth of $\mathrm{SS} n$ \\
\hline$M R R_{j}^{r e a}$ & $\begin{array}{l}\text { The minimum required bandwidth of the jth } \\
\text { connection }\end{array}$ \\
\hline$M L T R_{j}^{\text {rea }}$ & $\begin{array}{l}\text { The bandwidth that is needed for completely } \\
\text { transmitting all the packets before deadline by } \\
\text { the jth connection }\end{array}$ \\
\hline$S I N R_{n}$ & The SINR value of SS $n$ \\
\hline$M C S_{n}$ & The MCS adopted by SS $n$ \\
\hline$F_{n}$ & $\begin{array}{l}\text { The code rate of the error correction code used } \\
\text { by SS } n\end{array}$ \\
\hline $\mathrm{GER}_{\mathrm{n}}$ & The packet loss rate of SS $n$ \\
\hline Threshold loss_rate & The required packet loss rate by WiMAX \\
\hline
\end{tabular}

best solution in terms of minimum resource (time slots) requirements. In the second phase, two-stage scheduling was proposed to maximize the network throughput. The first-stage scheduling algorithm was used to determine the factors which allowed the system to reach load balancing while the aggregated network throughput is maximized by the second-stage scheduling.

\section{Proposed algorithms}

\subsection{MFSA}

Based on the various MCSs that were selected, the transmission rate and packet error rate also varied. Specifically, when a high data rate MCS (such as 64QAM) is selected by BS for transmitting packets to SS, fewer time slots are consumed when transmitting, but higher packet loss rate could be encountered. On the other hand, if a low data rate modulation (such as BPSK) is selected by BS for transmitting information to SS, then more time slots are consumed, but the packet loss rate would decline. Gopala and Gamal [20] therefore introduced the concept of opportunistic transmission: if the AL-FEC was used to help reduce the packet loss rate, then the BS could employ a better modulation to transmit the packet. As a consequence, for a system with $M$ MCSs and $F$ AL-FECs, the BS was offered $M^{*} F$ possible combinations of MCS and AL-FEC to choose from when transmitting packets to an SS. The objective of opportunistic transmission was to select the best combination which could minimize the consuming of time slots while satisfying the packet loss rate guarantee. In this section, we proposed a simple algorithm to achieve this goal.

First we introduced the equations for calculating the Group Error Rate (GER). Wang et al. [9] presented equations (11)-(14) for calculating the packet loss rate (PER) given the signal strength, represented by its SINR, and the selected MCS scheme. In Equations (11) and (12), SLER denotes the slot error rate; $L$ is the number of time slots used, $A$ and $B$ the constants which are chosen to fit the selected MCS curve for the given channel model. If a FEC, such as Reed-Solomon code, with code rate $k / n$ is adopted to reduce the packet error rate, then $k$ packets are encoded into $n$ packets so that receiving any $k$ or more packets of the transmitted $n$ packets could recover the original $k$ packets [11]. Thus, the error rate of a group of $k$ packets can be expressed by Equation (15).

$$
\begin{gathered}
e=1-(1-S L E R)^{L} \\
\text { SLER }=10^{A C_{m}+B} \\
C_{m}=\log _{2}\left(1+S_{N} R_{m}\right) \\
\operatorname{SINR}_{m}(d B)= \\
\quad S N R\left(f, d, H_{B S}, H_{s s}\right)+M d-S d_{m} \\
\quad-F d_{m}\left(f, s, n_{s c}\right)-I_{m} \\
\operatorname{GER}(\operatorname{SINR}, M C S, F E C)=\sum_{i=n-k+1}^{n} e^{i}(1-e)^{n-i}
\end{gathered}
$$

In this study, we proposed the MFSA, as shown in Figure 2, to determine the optimal combination of MCS and FEC which will achieve the smallest number of time slots while the GER meeting the threshold value

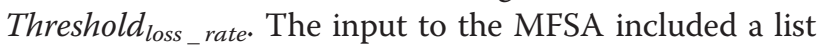
of selected SINR levels, the number of bits carried by each kind of MCS per time slot, and the code rate of FEC. The output of MFSA included the maximum number of bits per time slot the system was capable of carrying under each SINR level and the combination of MCS and FEC that was selected to achieve this maximum throughput. We assumed there are $D$ levels of SINR, $M$ possible MCS schemes, and $F$ kinds of FEC code rates. Three nested for loops enumerate $D^{*} M^{*} F$ possible combinations of SINR levels, modulation schemes, and FEC code rates. Within the nested for loop, line 9 checks if the GER was below the threshold. If it was below the threshold, the number of bits that the combination was capable of carrying was equal to the number of bits carried by MCS multiplies the FEC code rate. On the other hand, if the GER exceeds the threshold, then the number of bits that can be carried was zero. Lines 11-15 are to 


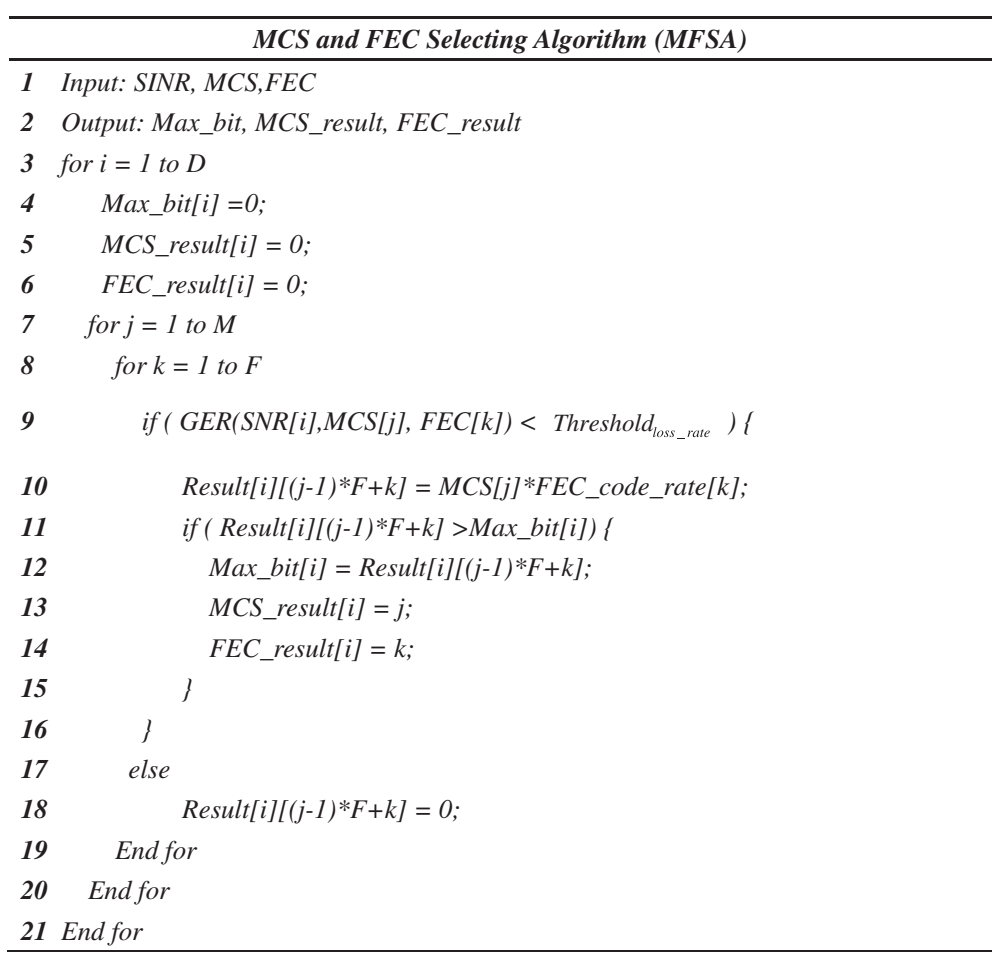

Figure 2 MFSA algorithm.

select and decide the best combination of MCS and FEC for $D$ SINR and obtain it as the output. The computation complexity of MFSA is $O\left(D^{*} M^{*} F\right)$. For the OLT to select the optimal combination of MCS and FEC for each SS, it first obtains the estimated SINR level of the SS from its associated ONU-BS and then selects the MCS and FEC based on the SINR level by looking up the output arrays of MFSA, namely MCS_result and FEC_result. It takes a constant to look up these two arrays.

\subsection{First-stage scheduling}

Because the number of SSs under the coverage of one ONU-BS varies and the total flow for each SS also varies, thus the load of each ONU-BS also varies. Therefore, in this section, we proposed a scheduling algorithm for forwarding packets from OLT to ONU-BS, referred to as the first-stage scheduling. The OLT will dynamically decide which ONU-BS will forward the packet of an OSS, an SS that are covered by the transmission range of two or more ONU-BSs, based on the least loaded policy. For example, if $\mathrm{OSS}_{n}$ could be served by either $\mathrm{ONU}_{-\mathrm{BS}_{i}}$ or ONU-BS${ }_{j}$. The OLT will dynamically select $\mathrm{ONU}_{-\mathrm{BS}_{i}}$ or ONU-BS ${ }_{j}$ as the serving BS for $\mathrm{OSS}_{n}$ according to their queue lengths so as to reduce the queuing delay. Suppose there are $p k t s_{-} B S_{i}$ packets in

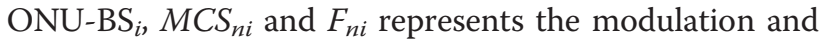
AL-FEC code rate used by $O S S_{n}$ to receive the packet, we now calculate the backlog of ONU-BS , which was the number of time slots required to finish transferring all $p k t s_{-} B S_{i}$ packets, by Equation (16):

$$
\text { backlog }_{i}=\sum_{j=1}^{p k t s \_B S_{i}} \frac{\text { packet_size }_{j}}{M C S_{n i} \times F_{n i}}
$$

When the information of the backlog of each ONU-BS is sent to the OLT via MPCP, the OLT can balance the load of ONU-BSs via forwarding packets to ONU-BSs based on their backlog. The First-Stage Scheduling algorithm, as shown in Figure 3, is proposed to achieve this goal. Initially, the algorithm sets the backlog of the current Original_BS as the minimum backlog. It then compares the minimum backlog with that of the BSs available for forwarding the packet. If a New_BS with a smaller backlog is found, then the algorithm sets the New_BS as the serving BS for all OSSs that are within the transmission range of the Original_BS and the New_BS; otherwise no action will be taken.

\subsection{Second-stage scheduling}

IEEE 802.16e supports five classes of traffic, namely, Unsolicited Grant Service (UGS), extend-real-time Polling Service (ertPS), real-time Polling Service (rtPS), non-real-time Polling Service (nrtPS), and best effort (BE) [21]. Therefore, at each ONU-BS, each class of traffic has its own queue and QoS requirement. Packet 


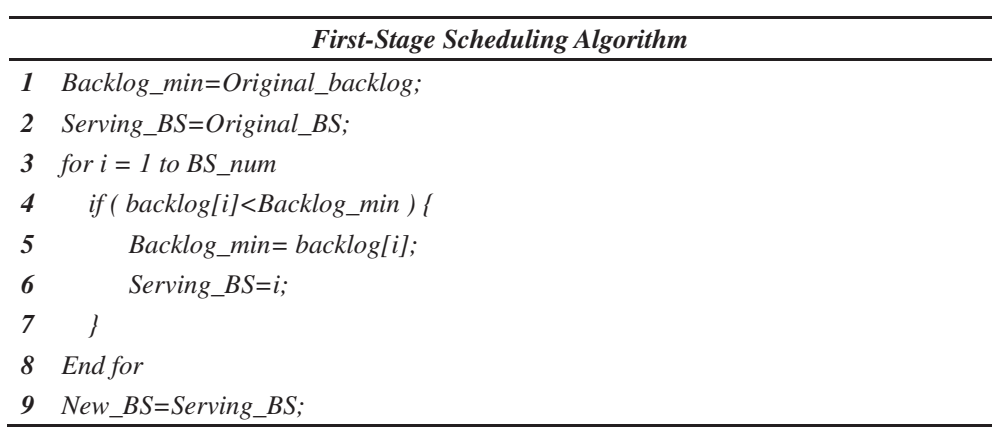

Figure 3 The First-Stage Scheduling algorithm.

scheduling at ONU-BS is thus required to guarantee the QoS of each traffic class. The most commonly adopted scheduling policy is based on strict priority which allocates bandwidth and transmits packets simply based on the priority of the traffic class with UGS having the highest priority and BE having the lowest priority. The advantage of strict priority scheduling was that services with real-time requirement were satisfied preferentially. But the disadvantage was that it is unfair for non-real time services and bandwidth starvation could occur. For example, nrtPS traffic might not be able to gain access to bandwidth, causing the QoS of nrtPS to not be guaranteed. Moreover, as the SINR of each SS is prone to change dynamically, allocating time slots of a WiMAX frame to high priority traffic, such as rtPS, may cause poor system throughput. The reason is that the modulation used by rtPS connection may improve in the next frame, the consumption of time slots will be reduced if the forwarding of packets to the rtPS connection was postponed till the next frame. We therefore proposed the following second-stage scheduling algorithm for ONU-BSs. First we calculated the Minimum Reserved Rate (MRR) and the Maximum Latency Tolerance Rate (MLTR) of five service types. The calculation was based on Equations (17) and (18).

$$
\begin{aligned}
& \operatorname{MRR}_{i}^{\text {req }}=\sum_{j \in i} R_{j}^{\text {req }} \\
& \operatorname{MLTR}_{i}^{\text {req }}=\sum_{j \in i} \sum_{k \in j} \frac{\text { packet_size }}{D_{\text {remain }}\left(\tau_{k}\right)}
\end{aligned}
$$

In Equations (17) and (18), $i$ denotes the type of service, $j$ the index of the connections of that service type, $k$ the index of packets in the queue of that connection, $M R R_{i}^{r e q}$ is the minimum required bandwidth of service type $i$, and $M L T R_{i}^{r e q}$ is the required bandwidth to meet the delay requirement of real time traffic (e.g., rtPS). Equation (17) calculated the minimum required bandwidth for each service type $i$. For example, the sum total of the MMR of rtPS connections was the MRR required for the service type of rtPS. Equation (18) calculated the required bandwidth for service types (such as rtPS) with delay requirement, where packet_size $k_{k}$ is the length of packet $k$ in the queue of connection $j$, in bits; $D_{\text {remain }}\left(\tau_{k}\right)$ was the remaining latency time with tolerable delay of packet $k$ in the queue of connection $j$, in seconds.

Since ertPS can be emulated by rtPS, we will combine these two traffic classes into one. In addition, UGS requires fixed amount of bandwidth and $\mathrm{BE}$ does not have QoS guarantee, thus, we focused on determining how much bandwidth the ONU-BS will allocate to rtPS and nrtPS. The proposed scheduling algorithm is based on $\mathrm{WF}^{2} \mathrm{Q}[10]$. $\mathrm{WF}^{2} \mathrm{Q}$ is used to determine which packet in the queue was to be transmitted preferentially at a certain time. Consequently, end-to-end bounded-delay and fair allocation of bandwidth can be achieved. Time slots of a WiMAX frame were allocated to each traffic class based on Equations (19)-(25).

$$
\text { Available_slot }{ }_{U G S}=\text { total_dl_slots }
$$

remaining_slot $_{U G S}=$ Available_slot $t_{U G S}-$ used_slot $_{U G S}$

Available_slot $r t P S=\frac{W_{r t P S}}{W_{r t P S}+W_{\text {nrtPS }}} \times$ remaining_slot $_{U G S}$

\section{Table 2 System parameters used in simulations}

\begin{tabular}{ll}
\hline System Parameters & Value \\
\hline EPON data rate & $1 \mathrm{Gps}$ \\
EPON frame duration & $2 \mathrm{~ms}$ \\
WiMAX system bandwidth & $10 \mathrm{Mhz}$ \\
WiMAX frame duration & $5 \mathrm{~ms}$ \\
WiMAX dl_ratio & 0.5 \\
Number of OLT & 1 \\
Number of ONU-BS & 2 \\
Number of SS & 11 \\
Number of connection & 55 \\
\hline
\end{tabular}


Table 3 Specification of traffic classes

\begin{tabular}{lllll}
\hline Class & $\begin{array}{l}\text { Connections } \\
\text { per SS }\end{array}$ & Traffic model & $\begin{array}{l}\text { Inter-arrival } \\
\text { time }\end{array}$ & $\begin{array}{l}\text { QoS } \\
\text { parameters }\end{array}$ \\
\hline UGS & 1 & CBR & $20 \mathrm{~ms}$ & Delay: $20 \mathrm{~ms}$ \\
rtPS & 2 & VBR (poisson) & $20 \mathrm{~ms}$ & $\begin{array}{l}\text { Delay: } 60 \mathrm{~ms} \\
\text { Min. reserve } \\
\text { rate: traffic rate }\end{array}$ \\
nrtPS & 1 & VBR (poisson) & $20 \mathrm{~ms}$ & $\begin{array}{l}\text { Min. reserve } \\
\text { rate: traffic rate }\end{array}$ \\
& & CBR & $20 \mathrm{~ms}$ & None \\
\hline
\end{tabular}

remaining_slot $_{r t P S}=$ Available_slot ${ }_{r t P S}-$ used_slot $_{r t P S}$

$$
\begin{aligned}
& \text { Available_slot }{ }_{n r t P S}=\frac{W_{n r t P S}}{W_{r t P S}+W_{n r t P S}} \times \text { remaining_slot }_{U G S} \\
& + \text { remaining_slot }_{r t P S} \\
& W_{r t P S}=\max \left(M R R_{r t P S}^{r e q}, M L T R_{r t P S}^{r e q}\right) \\
& W_{n r t P S}=M R R_{n r t P S}^{r e q}
\end{aligned}
$$

Suppose that the ONU-BS has total_dl_slots time slots available in one frame, as shown in Equation (19), it will preferentially allocate all the total_dl_slots time slots to UGS connections first. If there exists a remaining bandwidth remaining_slot ${ }_{U G S}$, it will determine the weights of rtPS according to Equation (21), and then allocate the remaining slot ${ }_{U G S}$ to $\mathrm{rtPS}$ connections based on their weights. $W_{r t P S}$ is the maximum value of the minimum required bandwidth of rtPS and the delay tolerant required bandwidth, whereas the $\mathrm{W}_{\text {nrtPS }}$ is the minimum required bandwidth of nrtPS, as shown in Equations (24) and (25). If rtPS does not use up the bandwidth allocated to it, with remaining slot ${ }_{r t P S}$ remaining, then nrtPS connections will have available to them the bandwidth allocated to them based on their weights plus the remaining bandwidth not used up by rtPS, remaining slot ${ }_{r t P S}$, as shown in Equation (23). Finally, if nrtPS does not use up its bandwidth, then the ONU-BS will search out the SSs with better modulation and determine if there are packets in their rtPS and nrtPS connections that have not been transmitted. If there are such packets, then it will allocate the remaining bandwidth remaining_slot ${ }_{n r t P S}$ to the rtPS and nrtPS connections of the SSs with better modulation. Otherwise, it will allocate all the remaining bandwidth to the $\mathrm{BE}$ connections of all the SSs.

Upon receiving the allocated bandwidth, all the service types other than rtPS will commence scheduling according to the SINR values of their connections based on a greedy algorithm. That is, each traffic class will transmit preferentially packets to SSs which consume smallest number of time slots in order to increase the overall throughput. As for rtPS, it will commence scheduling according to the SINR values of its connections, then it will search the queue for packets which are about to fail to satisfy the delay guarantee. If there are such packets, it will allocate the bandwidth preferentially to them. Otherwise, it will transmit preferentially the packets which consume smallest number of time slots, just like the other service types.

\section{Numerical results}

\subsection{System parameters}

In this study, network simulator ns-2 (version 2.31) and the WiMAX model developed in [22] were used to simulate the EPON-WiMAX network. The simulated topology is shown in Figure 1, which consists of 1 OLT, 2 ONU-BSs and 11 SSs. Each SS has five concurrent connections $(\mathrm{UGS} \times 1, \mathrm{rtPS} \times 2, \mathrm{nrtPS} \times 1, \mathrm{BS} \times 1)$. The OLT receives packets from the wired network and then forwards the packets to the designated ONU-BS by using the first-stage scheduling. Upon receiving the packets, the ONU-BS would then determine how to transmit packets to SSs by using the second-stage scheduling. The system parameters of the simulated network and QoS requirements of the four traffic classes are shown in Tables 2 and 3, respectively.

Figure 4 shows the Discrete-Time Markov Chain (DTMC) to simulate the Scenario that the modulation scheme adopted by an SS will change from time-to-time due to the variation of its SINR. The time unit is set to the frame time of the WiMAX network, i.e., 5ms. As

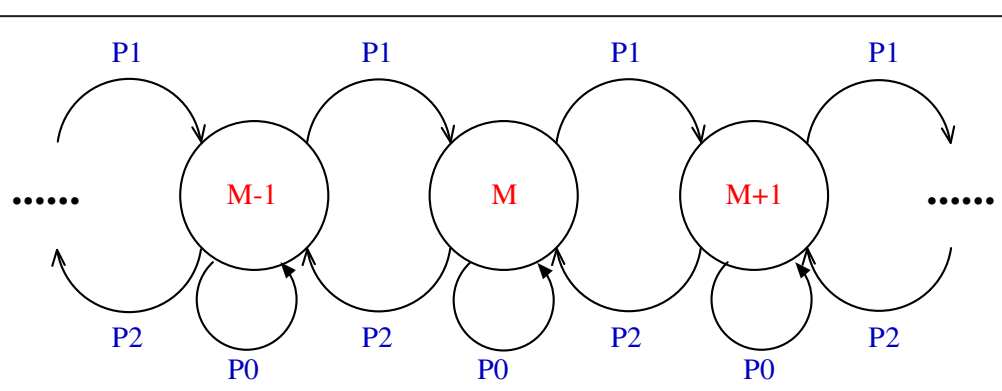

Figure 4 The DTMC model for simulating the dynamic change of MCS scheme. 
shown in Figure 4, if the $M$ th modulation scheme is adopted at current frame, the probability for staying unchanged is $P_{0}$, the probability for adopting a better modulation scheme (the $(M+1)$ th scheme) is $P_{1}$, and the probability of adopting a worse scheme (the $(\mathrm{M}-1)$ th scheme) is $P_{2}$. In our simulations, we set $P_{0}=0.6$, and $\mathrm{P}_{1}=\mathrm{P}_{2}=0.2$. For a system with eight MCSs, the steadystate probabilities are $\pi_{i}=0.125,0 \leq i \leq 7$.

\subsection{Numerical results of the proposed MFSA}

In this section, we show the numerical results of our MFSA algorithm. We set the GER threshold to $1.2^{*} 10^{-4}$. We assume that the system supports 8 MCS schemes, namely, BPSK, QPSK_1/2, QPSK_3/4, 16QAM_1/2, 16QAM_2/3, 16QAM_3/4,64QAM_2/3 and 64QAM_3/4. They are indexed from 0 to 7 where the higher index values the better data rates. The packet error rate, $e$, for each modulation scheme under various SINR is based on the reported simulation result of [19]. The SINR value received by an SS varies based on its distance from the serving ONU-BS. Table 4 shows the best MCS selected by MFSA under various distances without adopting the AL-FEC scheme. For example, if the SS is $0.1 \mathrm{~km}$ away from the service ONU-BS, 64QAM_3/4 is adopted and 27 bytes could be transmitted per time slot within a WiMAX frame.

Next, we consider adding the AL-FEC scheme to improve the transmission efficiency. We assume the maximum group block size, $n$, is 6 , and there are 16 code rates, ranging from $1 / 6$ to 1 where code rate $k / n$ denotes $k$ packets are encoded into $n$ packets. With AL-FEC, a better MCS with higher packet error rate could be selected because AL-FEC could recover lost packets. Table 5 shows the optimal combination of MCS and AL-FEC selected by the MFSA algorithm. From the data presented in Table 5, it can be observed that, when AL-FEC was applied, better modulation could be used while the GER could still be guaranteed. As a consequence, more bytes could be transmitted during one time slot. The bold values in Tables 4 and 5 showed that better data rate could be achieved, under different distances, when AL-FEC was adopted. For example, when an SS is $0.3 \mathrm{KM}$ away from the serving ONU-BS, the MFSA selects 64QAM_2/3 as the modulation scheme and an AL-FEC with code rate of 5/6 which yields a data rate of 20 bytes per time slot. However, if AL-FEC was not applied, ONU-BS could only select the 16QAM_3/4

Table 4 The best MCS selected by MFSA without adopting AL-FEC

\begin{tabular}{llllllll}
\hline Distance $(\mathbf{k m})$ & $\mathbf{0 . 1}$ & $\mathbf{0 . 2}$ & $\mathbf{0 . 3}$ & $\mathbf{0 . 4}$ & $\mathbf{0 . 5}$ & $\mathbf{0 . 6}$ & $\mathbf{0 . 7}$ \\
\hline MCS & 7 & 7 & $\mathbf{5}$ & $\mathbf{4}$ & $\mathbf{4}$ & $\mathbf{3}$ & $\mathbf{2}$ \\
Bytes per slot & 27.0 & 27.0 & $\mathbf{1 8 . 0}$ & $\mathbf{1 2 . 0}$ & $\mathbf{1 2 . 0}$ & $\mathbf{9 . 0}$ & $\mathbf{6 . 0}$ \\
\hline
\end{tabular}

Table 5 The best combination of MCS and AL-FEC selected by MFSA

\begin{tabular}{llllllll}
\hline Distance $(\mathbf{k m})$ & $\mathbf{0 . 1}$ & $\mathbf{0 . 2}$ & $\mathbf{0 . 3}$ & $\mathbf{0 . 4}$ & $\mathbf{0 . 5}$ & $\mathbf{0 . 6}$ & $\mathbf{0 . 7}$ \\
\hline MCS & 7 & 7 & $\mathbf{6}$ & $\mathbf{7}$ & $\mathbf{7}$ & $\mathbf{6}$ & $\mathbf{5}$ \\
AL-FEC code rate $=(k / n)$ & $1 / 1$ & $1 / 1$ & $\mathbf{5 / 6}$ & $\mathbf{4 / 6}$ & $\mathbf{3 / 6}$ & $\mathbf{3 / 6}$ & $\mathbf{3 / 6}$ \\
Bytes Per Slot & 27.0 & 27.0 & $\mathbf{2 0 . 0}$ & $\mathbf{1 8 . 0}$ & $\mathbf{1 3 . 5}$ & $\mathbf{1 2 . 0}$ & $\mathbf{9 . 0}$ \\
\hline
\end{tabular}

scheme which only yields a data rate of 18 bytes per time slot.

\subsection{Simulation results and discussions}

The simulated topology is shown in Figure 1 where 11 SSs were to be served by 2 ONU-BSs. There were five SSs that can only be served by ONU-BS ${ }_{0}$; their distances from $\mathrm{ONU}-\mathrm{BS}_{0}$ were $0.7,0.6,0.5,0.4$, and 0.3 , respectively. Similarly, five SSs with the same distance distribution can only be served by ONU-BS ${ }_{1}$. However, there was one OSS which was within the transmission range of both ONU-BS ${ }_{0}$ and ONU-BS ${ }_{1}$ and its distance from both ONU-BSs was 0.7 . There were five connections for each SS. Two Scenarios were simulated where traffic rates of UGS and rtPS were both fixed; only the traffic rates of nrtPS under two Scenarios were set differently. Under Scenario 1, the traffic rates were set such that the whole network was abundant if load balancing among two ONU-BSs was considered. That is, if the first-stage scheduling was applied, the load balancing was achieved through dynamic handover mechanism, and then the bandwidth requirements of UGS, rtPS and nrtPS were satisfied. But if the load balancing was not achieved through dynamic handover mechanism, then the bandwidth requirement of nrtPS could not be satisfied. For Scenario 2, the traffic rates were set much larger such that the bandwidth of the whole network was able to satisfy the bandwidth requirements of UGS and rtPS but was not able to satisfy the bandwidth requirement of nrtPS. The detailed flow parameters for each service type are shown in Table 6.

We compared four methods under these two Scenarios. The first method, denoted by the original, represents the baseline method in which a two-stage scheduling and AL-FEC were not applied. The second method, referred to as 1s_sch, adopts the first-stage scheduling

Table 6 Traffic parameters for each traffic class

\begin{tabular}{llll}
\hline Class & Traffic rate & Packet size & $\begin{array}{l}\text { Inter-arrival } \\
\text { time }\end{array}$ \\
\hline UGS & $64 \mathrm{kbps}$ & 160 bytes & $20 \mathrm{~ms}$ (CBR) \\
rtPS & $176 \mathrm{kbps}$ & 440 bytes & $20 \mathrm{~ms}$ (VBR) \\
nrtPS & Scenario 1: $232 \mathrm{kbps}$ & Scenario 1: 580 bytes & $20 \mathrm{~ms}$ (VBR) \\
& Scenario 2: $352 \mathrm{kbps}$ & Scenario 2: 880 bytes & \\
BE & $360 \mathrm{kbps}$ & 900 bytes & $20 \mathrm{~ms}$ (CBR) \\
\hline
\end{tabular}




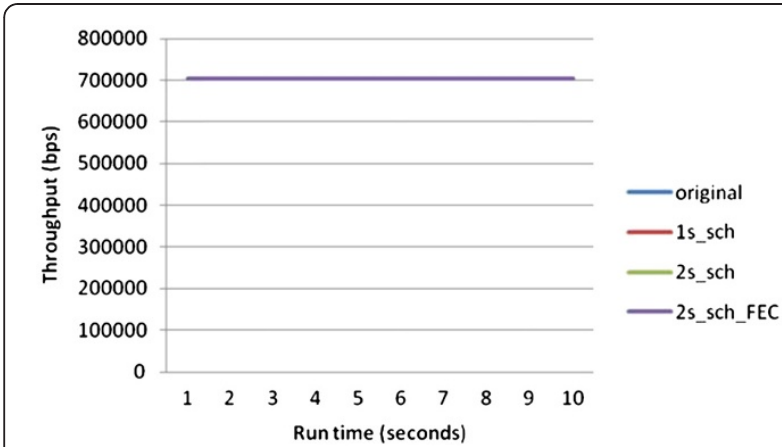

Figure 5 Throughput of UGS under Scenario 1 and 2.

such that the OLT would, based on the loads of both ONU-BSs, dynamically transmit the packets to the OSS via the ONU-BS with a smaller load. The third method, referred to as 2s_sch, adopts both the first-stage and second-stage scheduling algorithms at the OLT and ONU-BS, respectively. Lastly, the fourth method, denoted by 2s_sch_FEC, adopted MFSA and two-stage scheduling algorithms so that best combination of MCS and AL-FEC was selected and load balancing among ONU-BSs as well as $\mathrm{WF}^{2} \mathrm{Q}$ were applied to achieve the best system throughput.

\subsubsection{UGS traffic}

First, we investigate the performance of UGS traffic under the two Scenarios. Figure 5 represents the throughput of the UGS traffic provided by various scheduling methods under both Scenarios 1 and 2. Since ONU-BS allocates bandwidth preferentially to UGS every $20 \mathrm{~ms}$, the throughput of UGS was able to meet the required bandwidth under both Scenarios no matter which method is used. Figure 6 showed the delay of UGS yielded by various scheduling methods. Again, since ONU-BSs can allocate sufficient bandwidth to UGS traffic every $20 \mathrm{~ms}$, the delay of the UGS traffic was bounded to $20 \mathrm{~ms}$ which satisfies the delay constraint of the UGS traffic under every method in both Scenarios.

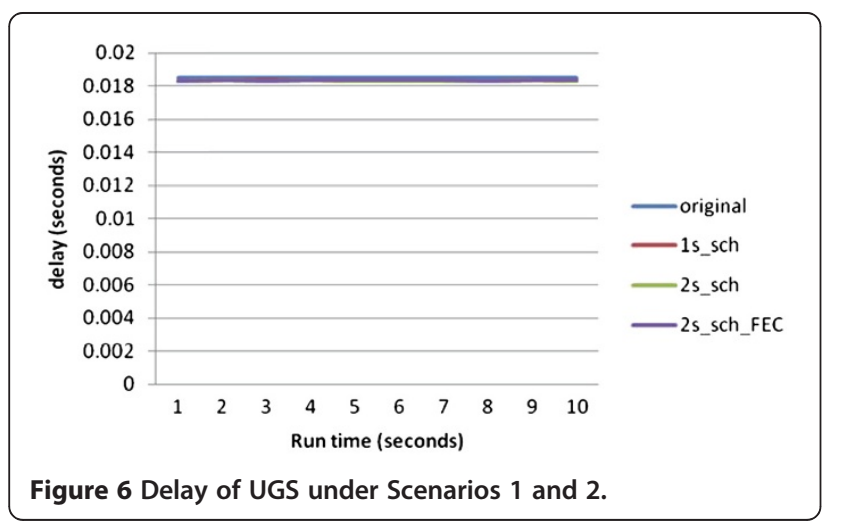

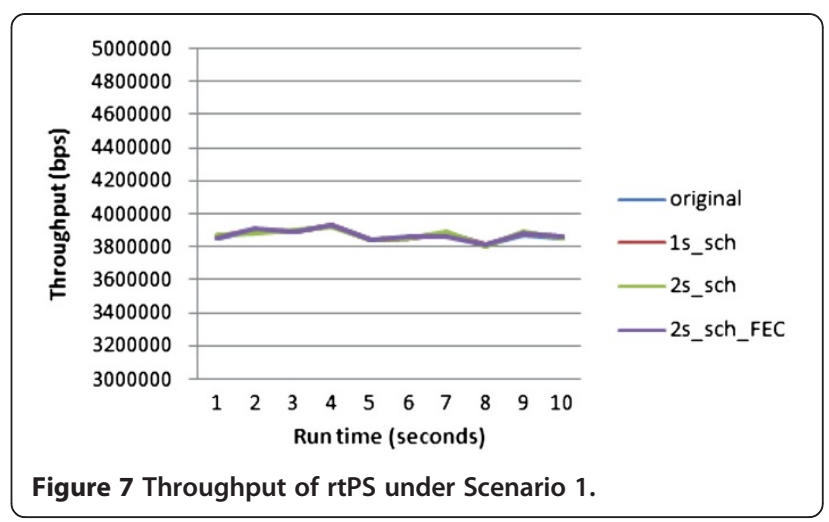

\subsection{2. rtPS traffic}

Figure 7 and 8 represent the throughput of rtPS under Scenarios 1 and 2, respectively. As the bandwidth of ONU-BS was sufficient to satisfy the bandwidth requirement of the rtPS traffic, upon finishing allocating bandwidth to UGS the ONU-BS would allocate bandwidth preferentially to rtPS traffic, so the throughput of rtPS traffic could achieve its requested rate in both Scenarios.

Figure 9 represents the delay time of the rtPS traffic under Scenario 1. Without the second-stage scheduling, both the original and 1s_sch methods allocate the bandwidth to rtPS preferentially after allocating bandwidth to the UGS traffic. As a consequence, the delay of rtPS under these two methods was bounded to $5 \mathrm{~ms}$, a WiMAX frame time. In other words, rtPS packets under these two methods are transmitted within one frame time. On the other hand, the $2 s \_s c h$ method adopts $\mathrm{WF}^{2} \mathrm{Q}$ and transmits packets of rtPS and nrtPS based on their weights. As a result, without violating the delay constraint of rtPS, a certain number of time slots would be allocated to nrtPS, rather than allocating all the time slots to rtPS connections with poorer modulation. Using the method, more time slots could be saved for transmitting the BE traffic. Note that the delay constraint of rtPS is set to $60 \mathrm{~ms}$, but the observed delay in Figure 9 was within 10ms. Thus, the QoS of rtPS was satisfied.

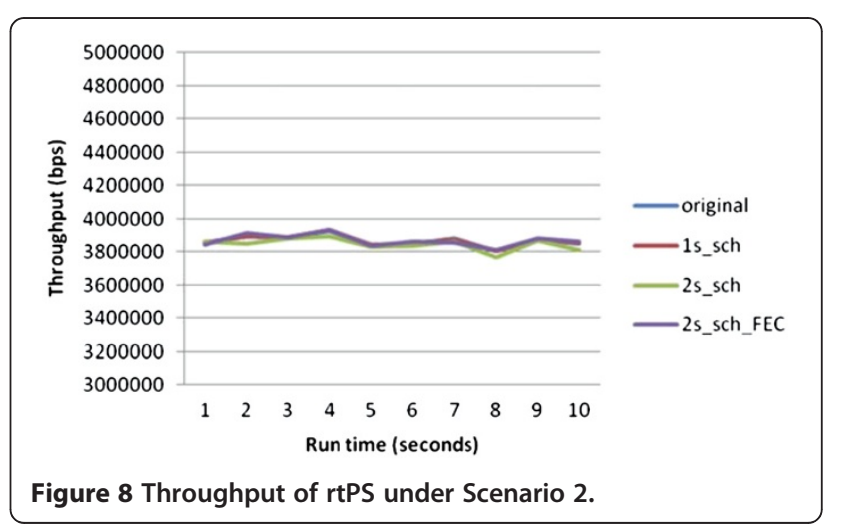




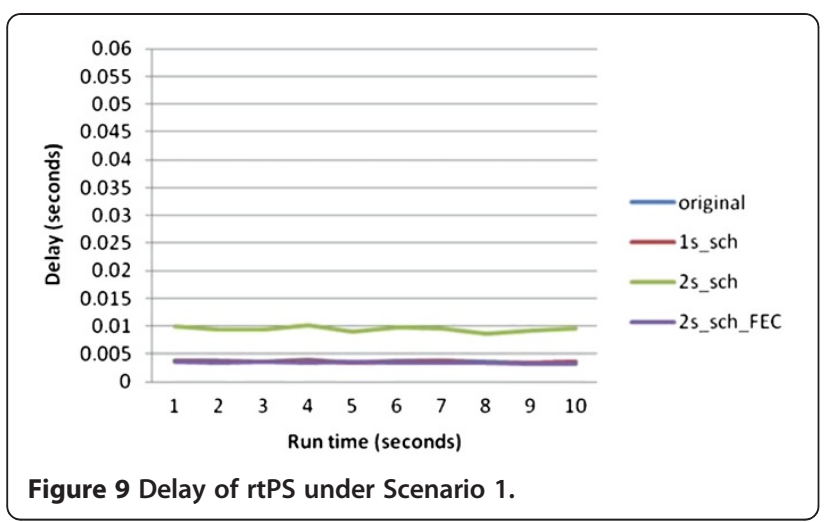

Finally, although the 2s_sch_FEC method also adopted the second-stage scheduling, but because AL-FEC was applied, the number of time slots which would be consumed by ONU-BS within each frame would be smaller than those methods without AL-FEC. Therefore, the bandwidth allocated to rtPS under the 2s_sch_FEC method was sufficient to transmit all of the rtPS packets within one WiMAX frame. As a consequence, the delay of rtPS under the 2s_sch_FEC method was bounded to $5 \mathrm{~ms}$.

Figure 10 represents the delay of rtPS which was similar to that of Figure 9. Same rationale of Figure 9 also applies here. However, since the bandwidth under Scenario 2 was not sufficient to meet the required data rates of both rtPS and nrtPS, thus the delay of rtPS yield by $2 s \_s c h$ was greater than that in Scenario 1 . But the delay was still below $20 \mathrm{~ms}$, which was less than $60 \mathrm{~ms}$, the delay constraint required by rtPS. Finally, delay yielded by $2 s \_s c h \_F E C$ was also very low. As we discussed in Figure 7 and 8, this was due to efficient usage of time slots. Therefore, the bandwidth received by rtPS, as allocated to them based on their weights at the beginning of the second-stage scheduling, would be sufficient to satisfy the bandwidth requirement of rtPS traffic, and the delay time is thus less than $5 \mathrm{~ms}$.

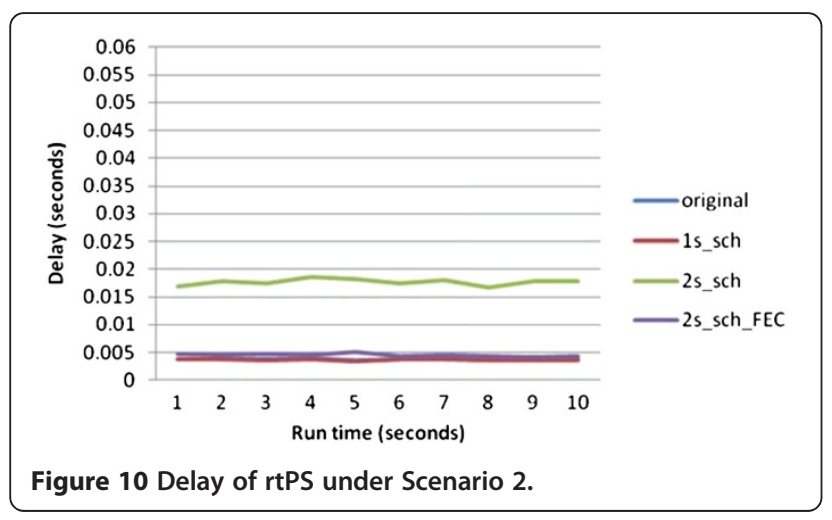

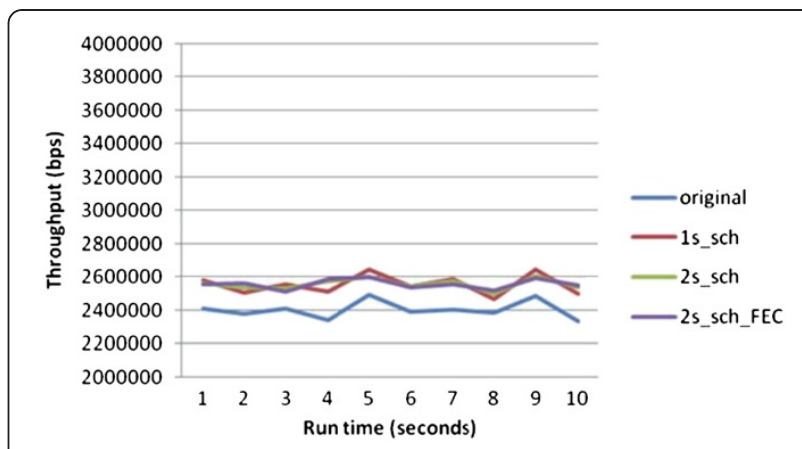

Figure 11 Throughput of nrtPS under Scenario 1.

\subsection{3. nrtPS traffic}

Figure 11 represents the throughput of nrtPS under Scenario 1 . Since the first-stage scheduling was not adopted in the original method, the OLT was not able to balance the load between ONU-BS $S_{0}$ and $\mathrm{ONU}-\mathrm{BS}_{1}$. As a consequence, $\mathrm{ONU}-\mathrm{BS}_{0}$ became overloaded and, thus, was not able to provide sufficient bandwidth to the nrtPS traffic. As can be observed from Figure 11, the throughput of nrtPS of the original method was lower than that of the other three methods which adopted the first-stage scheduling. In other words, the proposed first-stage scheduling was able to achieve load balancing and provide higher system throughput. In Figure 12, notice that the throughput of nrtPS in $2 s \_s c h$ was raised. This was due to the fact that under Scenario 2 the bandwidth of the whole system could not satisfy both rtPS and nrtPS sufficiently, thus during second-stage scheduling, the ONU-BS would allocate the available bandwidth to rtPS and nrtPS based on their weights, rather than allocating bandwidth to guarantee the minimum bandwidth requirement of rtPS only.

As shown in Figure 13, upon finishing the first-stage scheduling, if the second-stage scheduling was not applied, then $\mathrm{rtPS}$ would receive $100 \%$ of its minimum bandwidth requirement, whereas nrtPS would only receive $83.4 \%$ of its minimum bandwidth requirement.

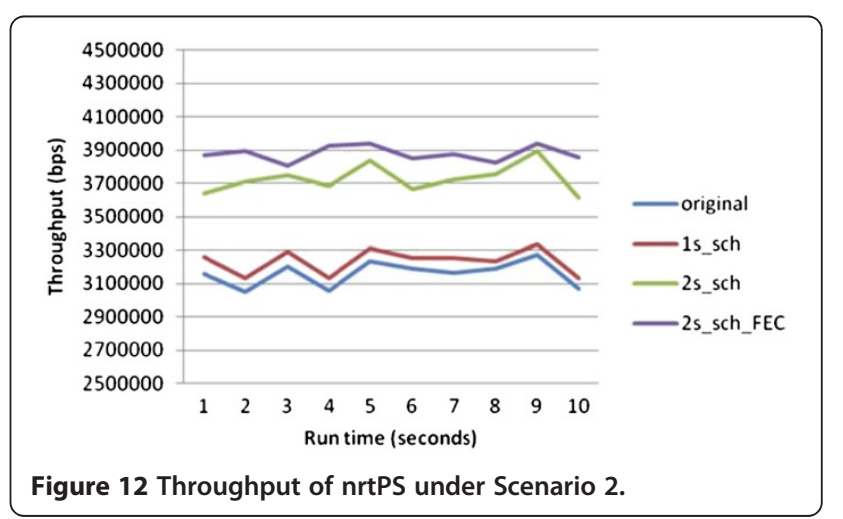




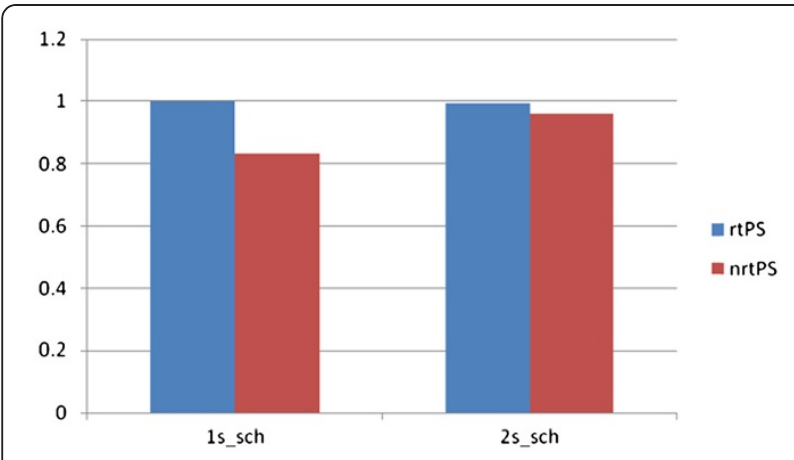

Figure 13 The percentage of allocated bandwidth to the required bandwidth of rtPS and nrtPS under $1 s \_s c h$ and $2 s \_s c h$ schemes in Scenario 2.

If both stages of the scheduling were applied, rtPS would satisfy $99.5 \%$ of its minimum bandwidth requirement, whereas nrtPS would also satisfy $96.1 \%$ of its minimum bandwidth requirement. In addition, when AL-FEC was adopted, as the number of time slots consumed was reduced, the bandwidth was sufficient to satisfy the minimum bandwidth requirements of both rtPS and nrtPS.

\subsubsection{BE traffic}

Figures 14 and 15 display the throughput of the BE traffic under Scenarios 1 and 2, respectively. From this figure, we can clearly observe that adopting AL-FEC increased the throughput of BE significantly. This observation was consistence with the literature, such as [19]. Besides, we can also observe that by transmitting packets to SSs with better modulation, the second-stage scheduling also improved the throughput of the BE traffic without decreasing the throughput of rtPS and nrtPS. The rationale was that by transmitting packets to SSs with better modulation, more time slots could be saved for transmitting more $\mathrm{BE}$ traffic. Because almost all the available bandwidth had been used to guarantee the QoS of UGS, rtPS and nrtPS traffic, the throughput of BE yielded by various scheduling methods without adopting AL-FEC differed insignificantly, as shown in Figure 15.

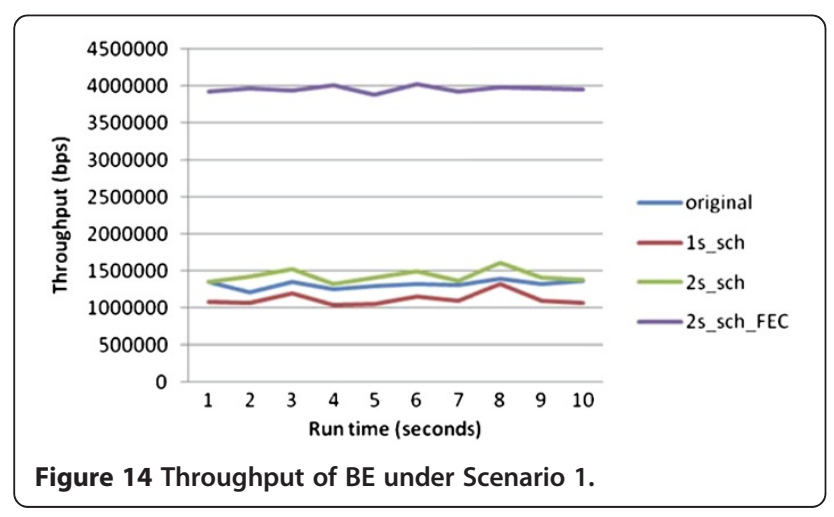

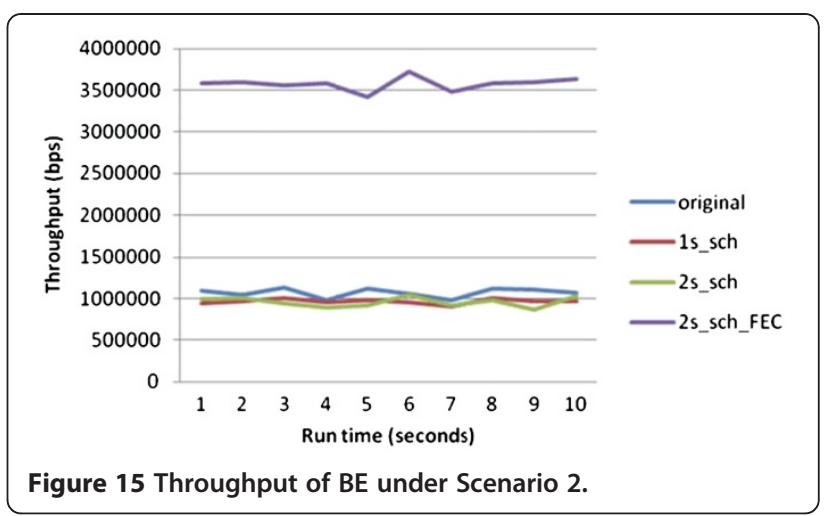

However, when FEC was applied, the throughput of BE was improved significantly.

\subsubsection{The Overall System throughput}

Lastly, the overall system throughput, which was the sum total of the throughput of UGS, rtPS, nrtPS and BE, under the two Scenarios were compared. Figures 16 and 17 showed the overall system throughput under Scenarios 1 and 2, respectively. It was obvious from the figures that the system throughput was higher in the methods where the two stage scheduling was adopted than in the methods where it was not adopted. Furthermore, when AL-FEC was applied, due to the reduced consumption of time slots, the system throughput could be improved even much greater.

\section{Conclusion}

EPON-WiMAX is a promising solution for broadband access network with mobility support. In this study, we focused on the downlink scheduling problem from OLT to ONU-BS, referred to as the first-stage scheduling, and from ONU-BS to SSs, referred to as the second-stage scheduling. In the first-stage scheduling, the OLT tried to balance the traffic load of ONU-BSs based on their queue length. In the second-stage scheduling, $\mathrm{WF}^{2} \mathrm{Q}$ was used to determine which packet in the queue was to

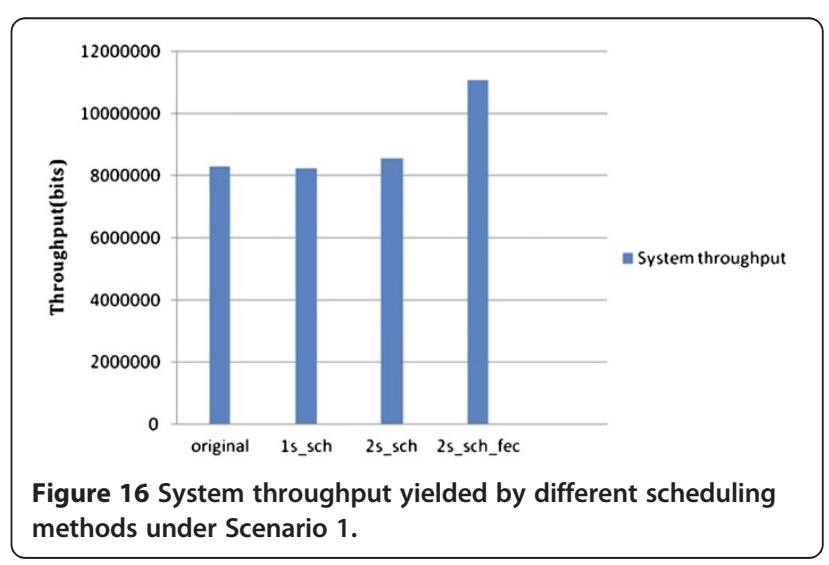




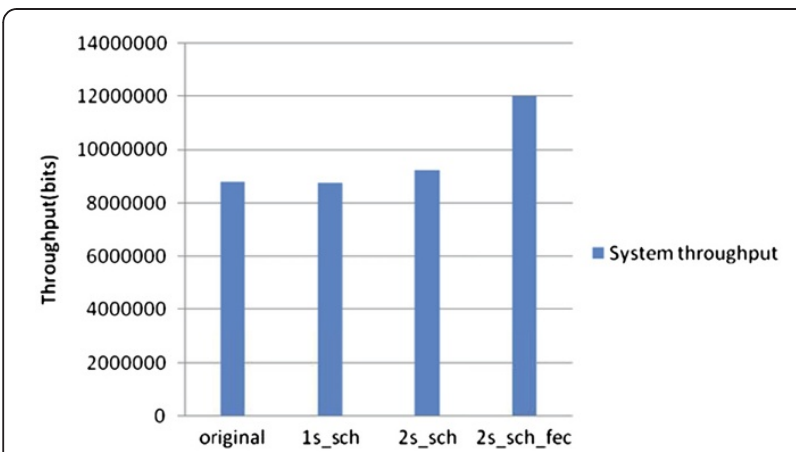

Figure 17 System throughput yielded by different scheduling methods under Scenario 2.

be transmitted preferentially at a certain time. We have also proposed the MFSA algorithm to determine the optimal combination of modulation and AL-FEC would be used for receiving packets by SSs under various SINRs so that the resource (time slots) consumption could be minimized. Our simulation results have shown the effectiveness of the two-stage scheduling, MFSA, and the applied AL-FEC. Specifically, these schemes were able to improve the system throughput significantly while guaranteeing the QoS of different traffic class.

In this study, we assumed that one SS would only receive packets from one BS at any one moment. In future studies, we will be more focused on studying the cooperative transmission among multiple BSs by using Space-Time Coding [23], such that the system throughput could be more enhanced.

\section{Competing interests}

The authors declare that they have no competing interests.

\section{Acknowledgements}

The authors would like to thank the National Science Council of the Republic of China, Taiwan for financially supporting this research under Contract No. NSC 100-2221-E-194-012-MY3 and NSC 100-2221-E-194-027-MY3.

Received: 14 June 2012 Accepted: 20 October 2012

Published: 2 November 2012

\section{References}

1. G. Kramer, G. Pesavento, Ethernet passive optical network (EPON): building a next-generation optical access network. IEEE Commun Mag 40(2), 66-73 (2002)

2. IEEE 802.16, IEEE Standard for Local and Metropolitan Area Networks Part 16: Air Interface for Fixed Broadband Wireless Access Systems (2004)

3. IEEE 802.16e, IEEE Standard for Local and Metropolitan Area Networks Part 16: Air Interface for Fixed and Mobile Broadband Wireless Access Systems (2005)

4. G. Shen, R.S. Tucker, C.-J. Chae, Fixed Mobile Convergence Architectures for Broadband Access: Integration of EPON and WiMAX. IEEE Commun Mag 45(8), 44-50 (2007)

5. IEEE Std 8023ah, Ethernet in the First Mile (2004)

6. K. Yang, S. Ou, G. Ken, H.-H. Chen, Convergence of Ethernet PON and IEEE 802.16 Broadband Access Networks and its QoS-Aware Dynamic Bandwidth Allocation Scheme. IEEE J Selected Areas Commun 27(2), 101-116 (2009)

7. B. Jung, J.Y. Choi, Y.-T. Han, M.-G. Kim, M. Kang, Centralized Scheduling Mechanism for Enhanced End-to-End Delay and QoS Support in
Integrated Architecture of EPON and WiMAX. J Lightwave Technol 28(16), 2277-2288 (2010)

8. M. Gong, B. Lin, P.-H. Ho, BU association and resource allocation in integrated PON-WiMAX under inter-cell cooperative transmission (Proceedings of the 2011 IEEE International Symposium on Wireless Pervasive Computing, Hong Kong, China, 2010), pp. 606-611

9. J. Wang, M. Venkatachalam, Y. Fang, System Architecture and Cross-Layer Optimization of Video Broadcast over WiMAX. IEEE J Selected Areas Commun 25(4), 712-721 (2007)

10. J. Bennett, H. Zhang, "WF2Q: Worst case fair weighted fair queuing", Proceedings of IEEE INFOCOM'96 (California, San Francisco, 1996), pp. $120-126$

11. M. Luby, T. Stockhammer, M. Watson, Application Layer FEC in IPTV Services. IEEE Commun Mag 45(5), 94-101 (2008)

12. N. Ghazisaidi, M. Maier, Techno-economic analysis of EPON and WiMAX for future Fiber-Wireless (FiWi) networks. Comput Netw 54(15), 2640-2650 (2010)

13. B. Lin, P.-H. Ho, X. Shen, F.C.-W. Su, Network Planning for Next-Generation Metropolitan-Area Broadband Access under EPON WiMAX Integration, Proceedings of IEEE Globecom 2008 (New Orleans, LA, USA, 2008), pp. 1-5

14. M.-G. Kim, G. Shen, J. Choi, B. Jung, H.-S. Park, M. Kang, Distributed antenna-based EPON WiMAX integration and its cost-efficient cell planning. IEEE J Selected Areas Commun 28(6), 808-817 (2010)

15. C. Ranaweera, E. Wong, C. Lim, A. Nirmalathas, Quality of service assurance in EPON WiMAX converged network, Proceedings of the 2011 IEEE International Topical Meeting on Microwave Photonics - Jointly Held with the 2011 Asia-Pacific Microwave Photonics Conference (Singapore, Singapore, 2011), pp. 369-372

16. M. Dias Piquet, N.L. Saldanha Fonseca, Performance Evaluation of a Scheduler for the ONU-BS of Integrated EPON WiMAX Networks. IEEE Lat AmTrans 10(3), 1838-1843 (2012)

17. J. She, P.-H. Ho, Cooperative Coded Video Multicast for IPTV Services under EPON WiMAX Integration. IEEE Commun Mag 46(8), 104-110 (2008)

18. S.K. Ray, K. Pawlikowski, H. Sirisena, Handover in Mobile WiMAX networks: the state of art and research issues. IEEE Commun Surv Tutorials 12(3), 376-399 (2010)

19. C.-W. Huang, S.-M. Huang, P.-H. Wu, S.-J. Lin, J-N. Hwang, OLM: Opportunistic Layered Multicasting for scalable IPTV over mobile WiMAX IEEE Trans Mobile Comput 11(3), 453-463 (2012)

20. P.K. Gopala, H. Gamal, "Opportunistic multicasting,", in Asilomar Conference on Signals, Systems and Computers 1, 2004, pp. 845-849

21. B. Li, Y. Qin, C.P. Low, C.L. Gwee, A Survey on Mobile WiMAX. IEEE Commun Mag 45(12), 70-75 (2007)

22. The WiMAX Forum System Level Simulator (, 2009). http://www.nist.gov/it// antd/emntg/ssm_seamlessandsecure.cfm

23. V. Tarokh, N. Seshadri, A.R. Calderbank, Space-time codes for high data rate wireless communication: performance criterion and code construction. IEEE Trans Inf Theory 44(2), 744-764 (1998)

doi:10.1186/1687-1499-2012-333

Cite this article as: Teng et al: Downlink packet scheduling and resource allocation in EPON WiMAX hybrid access networks. EURASIP Journal on Wireless Communications and Networking 2012 2012:333.

\section{Submit your manuscript to a SpringerOpen ${ }^{\odot}$ journal and benefit from:}

- Convenient online submission

- Rigorous peer review

- Immediate publication on acceptance

- Open access: articles freely available online

- High visibility within the field

- Retaining the copyright to your article

Submit your next manuscript at $\gg$ springeropen.com 